\title{
The Role of Molecular Tools in Genetic Diversity Conservation of Indigenous Chicken Production, Tanzania: Short Communication
}

\author{
Edward Moto \\ Department of Biology, College of Natural and Mathematical Sciences, University of Dodoma, Dodoma, Tanzania
}

Email address:

eddymotto@yahoo.co.uk

\section{To cite this article:}

Edward Moto. The Role of Molecular Tools in Genetic Diversity Conservation of Indigenous Chicken Production, Tanzania: Short Communication. American Journal of Polymer Science and Technology. Vol. 6, No. 2, 2020, pp. 10-13. doi: 10.11648/j.ajpst.20200602.11

Received: February 10, 2020; Accepted: March 11, 2020; Published: August 27, 2020

\begin{abstract}
Molecular techniques refers to methods used in molecular biology, biochemistry, genetics and biophysics which involve manipulation and analysis of DNA and RNA. Molecular techniques can also be used in biology to study genetic diversity and variation. Genetic diversity refers to the total number of genetic characteristics in the genetic makeup of a species. It shows similarity and variation that exist between breeds of the same species. Genetic diversity serves as a way for populations to adapt to changing environment. An increase loss of genetic diversity has been observed in most of agricultural species and particularly chicken genetic resources are considered to be the most threatened. Selective breeding by humans have led to the creation of many breeds characterized by high productivity, leading to the displacement of local breeds and posing a threat to the survival of many native breeds. Genetic resources are the building blocks for chicken development. The relevance of genetic diversity conservation in chicken production cannot be overemphasized because genes play a great role in formation of breeds and species. With recent advances in molecular technology, a number of techniques for in-depth genome analysis and evaluation of genetic variation in different breeds of chicken have been developed.
\end{abstract}

Keywords: Genetic Diversity, Conservation, Indigenous Chicken, Genetic Resources

\section{Introduction}

Genetic diversity of local poultry breeds is the most genetic diversity of the poultry world and play a critical roles in least developed countries such as Tanzania and others. Local chicken is among of the poultry breeds which are well adapted to extensive and semi intensive husbandry production system, contrasting to the exotic breeds. Local chicken are reported to undergo evolution over million years which enabled them to apt and being well reformed to the native environment [2]. In Tanzania poultry breeds has been largely classified based on phenotypic appearance and morphological structures such as body size and coat colour and information evidence obtained from indigenous communities such as adaptation and feeding habit, which may be inadequate and ambiguous [9]. Most of local chicken breeds are losing valuable genetic materials as they continue to undergo genetic diversity and variation. Regarding the conservation of animal genetic resources, whether in-situ or ex-situ, so far little efforts have been put in conserving the indigenous breeds. A report by the Ministry of Livestock Development and Fisheries (MLDF, 2013) reported that Tanzania has $60 \%$ of local chickens breeds, the rest $40 \%$ are exotic breeds. Thus, if there will be no intervention the exotic breeds could put the local breeds at risk of loss and under threat of extinction. Same scenario has been also reported in Tunisia by Ben Larbi et al., [2] in which about $30 \%$ of avian breeds are at high risk of loss and under threat of disappearance. Conservation strategies are very important in preservation of genetic constituents which are essential for evolution of species. Species that have narrow genetic variation are at a higher risk of extinction. Researchers have recognized that genetic variation is very important for maintaining biodiversity and evolutionary processes [6]. However, less has been done on global scale to protect loss of genetic diversity. In Tanzania, strains of indigenous chickens identified include Kawaida, Kishingo, Kinyavu, Kuchi and Bukini with the Kawaida having several ecotypes. The strains differ in body size, laying and survival rate. Currently there is a project at the Livestock Research 
Institute Mpwapwa where different strains of indigenous chickens are kept for the purpose of detailed characterization to establish whether the different strains are genetically different or just ecotypes. However, for the other strains of livestock species identified, it is important to carry out molecular characterization to establish whether they differ genetically. Presently molecular characterization is done at the Sokoine University of agriculture and the capacity of conducting DNA analysis is being built by establishing a biotechnology laboratory at the Tanzania Livestock Research Institute (TALIRI) Mpwapwa center.

Several studies on genetic characterization of indigenous chicken in Tanzania has been carried out, include; Lyimo et al., [7], characterized indigenous chicken in East and coast zone, Msoffe et al., [10] characterized chicken in Morogoro region and Moto and Rubanza [8] characterized indigenous chicken in the Central part of Tanzania. All those studies reported a great genetic variation of local chicken exists in Tanzania. However, genetic diversity conservation of animal in Tanzania, has remained largely a sheer task for academic institutes. Livestock research institutes have done very little in conservation of animal genetic including local chicken genetic. The aims of this manuscript is to show up the role of molecular tools in study of the genetic diversity conservation of traditional chicken breeds production in Tanzania.

\section{Discussion}

\subsection{The Loss of Genetic Diversity in Indigenous Chicken Breeds in Tanzania}

Conservation and utilization of genetic resources are constrained by the low genetic potential for production, inadequate documented information on productivity performance, lack of rights of the people who developed and maintained them, indiscriminate use and crossing of the resources and non-existence of livestock breeders associations and societies. In Tanzania, the decline in local chicken genetic diversity and genetic variation are caused by various factors including.

\subsection{The Substitution of Local Breed by Exotic Breeds}

Introduction of exotic breeds for the purpose of improving local chicken breeds in term of growth weigh, increase number of laid eggs and carcass weight has resulted into decline of indigenous chicken breeds. The demand of chicken protein has resulted into establishment of commercial breeds which are now used as breeding stocks to improve local chicken. The exotic and their crosses have been introduced in rural areas for the same purpose but in turn it is affecting the indigenous chicken which are well adapted to survive in their local environment.

\subsection{Indiscrimination of Crossbreeding}

Local chicken in low developed countries remain to be source of genetic diversity and variation characterized with low productivity. But demands for high production has resulted into development of different strategies in order to improve productivity. The direct use of exotic and crossbreeds as the way of improving local chicken has been used for several decades. Despite the failure of such strategy, but still is the common strategy. One of the major consequence of using exotic breeds or crossbreeding, is that it pose a threat for the disappearance of local genetic diversity, inducing displacement or genetic loss [4]. Unselective crossbreeding or extensive use of exotic germplasm can lead to genetic corrosion by dilution or extinction of the local genetic pool. This jeopardy may result into replacement of local genotypes with those of commercial crossbreeds, which have a greater production potential with high nutrient requirements but are not well adapted for survival in local environments [15].

\subsection{The Consumer's Preference}

Consumers demand due to certain provisions in chicken products has resulted into passionate genetic selection and breeding of the preferred breeds and lines in order to meet consumers demand. The intense genetic selection leads to loss of germplasm in indigenous chicken due to introduction of exotic commercial and industrial stocks. Narrowing genetic diversity make it difficult for some breeds to cope with extreme environmental challenges like disease outbreaks and climatic change. Achievement in commercial chicken breeding is dependent upon genetic composition of the populations and environmental interaction, while reduced variability may results in a limited flexibility to attain new breeding goals [2].

\subsection{The Importance of Conservation of Indigenous Chicken Genetic Diversity}

The indigenous chicken breeds are well adapted and suited to survive in harsh local environments which include low quality feeds, diseases outbreak and high environmental temperature. Nevertheless, in least developed countries like Tanzania some of indigenous chicken breeds are in danger of loss or being vanished due to unselective crossbreeding with other breeds and lack of proper and controlled mating. The significance of genetic diversity conservation in indigenous chicken production cannot be overemphasized because genes play a major role in formation of new breeds. Genetic resources are the building blocks for development of chicken breeds with desirable features and characteristics. It allows animal breeders and farmers to act and respond to dynamic environmental changes and to meet consumers' request. Conservation of indigenous chicken genetic diversity helps to lessen the reliance on exotic chicken breeds as the improvement strategy of local breeds is encouraged. In Tanzania, the livestock research centers existing in the country have started be used as source of breeding stock to support initiation of in-situ conservation and improvement of animal genetic resources within the allied communities. However, there is a need to develop capacities for undertaking both in-situ and ex-situ conservation programs by establishing infrastructure and training of human resources needed for the 
conservation, management and sustainable utilization of animal genetic resources, including experts in legal issues on patents and international collaboration.

Conservation of indigenous chicken play a great role in preserving those with desirable traits and produce chicken with high quality grades which can be used as the parent stock. Also it encourages survival of fittest through natural selection in which the better are able to deal with diverse and miscellaneous environmental challenges. Wikipedia (2013) reported that if a population has a very diverse gene pool, there will be great variation in the traits of the individuals in that population and subsequently more traits for natural selection to act upon the fittest individuals to persist and prevail.

Genetic diversity and biodiversity are reliant on upon each other for success of an individual. Genetic diversity within a species is indispensable to preserve diversity among species, and vice versa (NSF, 2006). The authors argued that if one element is eliminated from the system, the succession can break down, and the community may become dominated by a single species. The National Biological Information Infrastructure (2008) proclaimed that the interdependency between genetic and biological diversity may lead to changes in the environment, leading to adaptation of the remaining species; and changes in genetic diversity may leads to a loss of biological diversity [11]. As breeds continue to evolve through genetic diversity and variation, most of the desired genetic materials are vanished. Genetic conservation values support to preserve the genetic constituent of species which are essential for species to evolve and adaptation. Species that have low genetic variation are at a greater risk of extinction.

\subsection{Measuring of Genetic Diversity}

Conservation of genetic diversity of indigenous chicken resources at satisfactory levels requires application of systematic scientific knowledge and principles. This requires the integration of population genetics and molecular biology tools such as molecular markers.

Many chicken breeds have been described based on phenotypic qualities, not taking into consideration that large parts of the genomic make-up of these breeds might be the same. In order to have an effective and efficient conservation strategies, thus, reliable information about genetic difference between and among individuals, populations and breeds are required. Recently, characterization of poultry breeds have shifted from phenotypic and morphological features and emphasize has been on measurements at the molecular or genetic level [12].

\subsection{Measurement of Genetic Diversity by Phenotypic Approach}

The use of phenotypic traits gives the first insight into breed variation and diversity by investigating the differences exist in physical appearance. Phenotypic markers may be subdivided into discontinues traits and continuous traits that are applicable to assessing genetic variation and phylogenetic relationships between various breeds and populations. Several authors have applied this method to characterize and differentiate animals' breeds [9].

\subsection{Use of Molecular Markers in Measurement of Genetic Diversity}

The advance of technologies and particular molecular technology has enabled to study and characterize breeds at genetic level. Different classes of molecular markers has come into application in study of genetic variation among breeds. Among the molecular markers include; restriction fragment length polymorphism (RFLP), random amplified polymorphic DNA (RAPD) markers, amplified fragment length polymorphisms (AFLP), mitochondrial DNA (mtDNA) markers, microsatellites and minisatellites, as well as single nucleotide polymorphisms (SNP) markers have been applied in genetic diversity studies [15].

Assessment of gene polymorphism by the use of DNA marker proposes that variability in DNA is a powerful tool in scrutinizing diversity which exist within and among individuals, families and populations [14]. Studies have shown that molecular markers such as microsatellite marker can be used to examine pooled DNA samples for population studies [7, 8, 10, 13]. The studies by Msoffe et al., [10]; Moto and Rubanza [8] using 18 microsatellite sequences revealed that there is a great genetic variation within and between populations of local indigenous chicken in Tanzania. These could be a good starting point in conservation of indigenous chicken with desirable traits found in Tanzania. The preliminary findings obtained from these studies may also provide background for future studies of identification of the genetic uniqueness of Tanzanian native chicken breeds, breeding programmes and development of conservation strategies [8]. The evaluation of five ecotypes of chicken populations by Lyimo et al., [7] based on analysis of 29 microsatellite sequences revealed that Tanzanian chickens probably originated on the Indian subcontinent and in Southeast Asia. They were probably brought to Tanzania during colonial era. The use of microsatellite has been found to be very useful in studying genetic variation among populations and between individuals [15]. Furthermore, it has been also reported that, single nucleotide polymorphism (SNP) has been recognized as a very auspicious level of molecular markers which can be used to study biodiversity and to study diversity within and between breeds. The major principle of SNPs is to hybridize detected DNA fragments with high-density DNA probe arrays; the SNP allele is then termed according to the hybridization results [18]. Advance in modern biotechnologies will enable the dramatic expansion of the sequence information within species will afford completely new possibilities for the use of genome variability in the next years. Authors has also claimed that SNPs will allow evaluation of variation within coding regions of genes, and may therefore offer higher sensitivity compare to microsatellites for recognizing populations of greater conservation traits. The authors nevertheless pointed out that the appropriateness of SNPs as molecular markers in biodiversity studies needs to be properly assessed, since DNA polymorphism at the population level is affected by numerous constraints like the position in the genome, drift, selection and recombination. 
On the other hand, AFLPs have been referred as reproducible biallelic markers that are distributed through the genome as restriction sites, and they allow estimates of genetic differentiation between breeds and related species [1]. The AFLP markers have found the broadest application in analyses of genetic variation below the species level, predominantly in investigations of population structure and differentiation [5]. The presence or absence of amplification of specific fragments which correspond to insertion, deletion between restriction sites, or single nucleotide polymorphism at a site recognized by restriction enzymes can be analysed by AFLPs [13]. The time and cost efficiency, reproducibility and resolution of AFLPs are superior or equal to those of other markers such as RAPD, RFLP and microsatellites [3]. Zanetti [17] used AFLPs to determine the genetic diversity of local chicken breeds of north- eastern Italy.

\section{Conclusion}

The use of molecular markers have paved a way for researchers to study genetic diversity and variation in indigenous chicken between and among populations and breeds. The accurate evaluation of animals genetic is the major target for genetic diversity conservation and utilization. Different methods have been developed and tested at the DNA sequence level and opening up new opportunities for evaluating diversity in local chicken genetic resources. The information obtained are very useful in identifying breeds with potential traits which can be conserved and been used in breeding programmes and development. The knowledge of using molecular tools could be implemented and being used in animal research institutes to make use in conservation of animal genetic resources.

\section{Conflict of Interest}

I declare that no conflict of interest exists for this article.

\section{References}

[1] Ajmone-Marsan P., Negrini R., Milanesi E., Nijman I. J., Buntjer J. B., Valentini A., and Lenstra A. (2002). Genetic distances within and across cattle breeds as indicated by biallelic AFLP markers. Anim. Genet. 33: 280-286.

[2] Ben Larbi M., M'hamdi N., and Haddad B. (2013): Indigenous chicken production systems in villages in the south of Tunisia. Livestock Research for Rural Development. Volume 25, Article \#99. Retrieved December 12, 2019, from http://www.lrrd.org/lrrd25/6/larb25099.htm.

[3] Brumlop S., and Finckh, M. R. (2010). Applications and potentials of marker assisted selection (MAS) in plant breeding Final report of the F+E project "Applications and Potentials of Smart Breeding" (FKZ 350889 0020) On behalf of the Federal Agency for Nature Conservation December 2010.

[4] Hanotte O, Dessie T, Kemp S. (2010). Time to tap Africa's livestock genomes. Science. 2010; 328: 1640-1641. [PubMed] [Google Scholar].

[5] Hedrick P. (1992). Shooting the RAPDs. Nature 355: 679-680.

[6] Laikre, L. (2010). Genetic Diversity is overlooked in international conservation policy implementation. Conserv. Genet. 11: 349-354. DOI 10. 1007/S 10592-009-0037-4.

[7] Lyimo, C. M. A. Weigend, U. Janben-Tapken, P. L. Msoffe, H. Samianer, and Weigend, S. (2013). Assessing the genetic diversity of five Tanzanian chicken ecotypes using molecular tools, S. Afr. J. Anim. Sci. 4: 43.

[8] Moto E. and Rubanza C. K. (2019). Genetic diversity of local chicken ecotypes in selected part of Central Tanzania, Int. J. of Biosciences, 14, (4) 15-25, 2019; http://dx.doi.org/10.12692/ijb/14.4.15-25.

[9] Msalya G, Lutatenekwa D, and Chenyambuga SW. (2017). Possibilities of utilizing biotechnology to improve animal and animal feeds productivity in Tanzania - review of past efforts and available opportunities. J Dairy Vet Anim Res. 2017; 5 (5): 161-167. DOI: 10.15406/jdvar.2017.05.00155.

[10] Msoffe, P. L. M., Mtambo, M. M. A., Minga, U. M., Juul-Madsen, H. R. and Gwakisa, P. S. (2005). Genetic structure among local chicken ecotypes of Tanzania based on microsatellite DNA typing. Afr. J. Biotechnol. 4 (8), 768-771.

[11] Pullin, A. S. (2002). Conservation biology. Cambridge: Cambridge University Press. ISBN 9780521644822.

[12] Pym, R. (2013). Poultry genetics and breeding in developing countries. Genetic diversity of genetic resources. School of Veterinary Science, The University of Queensland, Gatton, 4343, Queensland, Australia.

[13] Semik E. and Krawczyk J. (2011). The state of poultry genetic resources and genetic diversity of hen populations. Ann. Anim. Sci. 11 (2): 181-191.

[14] Weigend S. and Romanov M. N. (2002). The world watch list for domestic animal diversity in the context of conservation and utilization of poultry biodiversity. Poultry Sci., 58 (4): 411-430.

[15] Weigend S., Romanov M. N. and Detlef R. (2013). Methodologies to identify, evaluate and conserve poultry genetic resources. Institute for Animal Breeding Mariensee of the Federal Agricultural Research Centre, 31535 Neustadt, Germany. Retrieved on 26/10/2019.

[16] Firas R. Al-Samarai, Abdulkareem A. Al-Kazaz (2015). Applications of Molecular Markers in Animal Breeding: A review, American Journal of Applied Scientific Research. Vol. 1, No. 1, pp. 1-5. Doi: 10.11648/j.ajasr.20150101.11.

[17] Zanetti E, De Marchi M., Dalvit C, Cassandro M (2010). Genetic characterization of local Italian breeds of chickens undergoing in situ conservation. Poultry Sci. 89: 420-427. 10.3382/ps.2009-00324 [PubMed] [CrossRef] [Google Scholar].

[18] Zhang F, et al. (2013). Genetic mapping of quantitative trait loci underlying flowering time in chrysanthemum (Chrysanthemum morifolium). PLoS One 8: e83023 [Google Scholar] [Crossref PubMed]. 